\title{
Microcephaly-capillary malformation syndrome
}

INSERM

\section{Source}

INSERM. (1999). Orphanet: an online rare disease and orphan drug data base.

Microcephaly-capillary malformation syndrome. ORPHA:294016

Microcephaly-capillary malformation syndrome is a rare, genetic vascular anomaly

characterized by severe congenital microcephaly, poor somatic growth, diffuse multiple capillary malformations on the skin, intractable epilepsy, profound global developmental delay, spastic quadriparesis and hypoplastic distal phalanges. 